\title{
Seroprevalence of human immunodeficiency virus among antenatal patients and incidence of mother- to- child transmission using single dose nevirapine: a cohort study in Maharashtra
}

\author{
Sonal A. Bhuyar*, Pratibha V. Kale
}

\begin{abstract}
Associate Professor, Department of Obstetrics \& Gynaecology, Dr. Panjabrao Deshmukh Memorial Medical College
\end{abstract} (PDMMC), Amravati, Maharashtra, India

Received: 06 August 2015

Accepted: 21 August 2015

\section{*Correspondence:}

Dr. Sonal A. Bhuyar,

E-mail: drsonalbhuyar14@gmail.com

Copyright: (C) the author(s), publisher and licensee Medip Academy. This is an open-access article distributed under the terms of the Creative Commons Attribution Non-Commercial License, which permits unrestricted non-commercial use, distribution, and reproduction in any medium, provided the original work is properly cited.

\begin{abstract}
Background: Objective was to study the seroprevalence of human immunodeficiency virus (HIV) in antenatal patients and to find out the incidence of mother to child transmission (MTCT) using single dose nevirapine (sdNVP) to mother- baby pairs in labour.

Methods: This prospective study was conducted in the department of Obstetrics \& Gynecology, Dr. PDMMC, Amravati, Maharashtra from February 2010 to May 2015. All pregnant women attending the antenatal clinic were subjected to pretest counseling and HIV testing after informed consent. The HIV +ve pregnant patients who opted for delivery at our hospital were included in the study. sdNVP was administered to mother- baby pairs according to NACO guidelines. Babies of these patients were followed up to 18 months of age for HIV testing.

Results: 12077 antenatal patients underwent pretest counseling. 11957 antenatal patients were tested for HIV of which 28 were detected positive. Therefore, the seroprevalence in this study was found to be $0.23 \%$. 23 patients delivered at our hospital during the study period and babies of 2 patients tested positive for HIV. Hence the incidence of MTCT was found to be $9.5 \%$ in our study.

Conclusions: Universal screening of pregnant patients for HIV and judicious use of PPTCT regimens can significantly reduce MTCT of HIV and prevalence of pediatric HIV infection. Single dose nevirapine use over last few years has successfully reduced MTCT, but our aim should be to reduce it further with more effective ART regimens for the seropositive antenatal patients and their babies.
\end{abstract}

Keywords: Seroprevalence of HIV, Mother- to- child transmission, Single dose nevirapine

\section{INTRODUCTION}

India has the third highest number of estimated people living with human immunodeficiency virus (PLHIV) in the world. According to HIV estimations 2012, number of PLHIV in India was 20.89 lakhs, of which 39\% were women with a national HIV prevalence for ANC attendees being $0.35 \%$. This feminization of the HIV epidemic in India has resulted in increasing number of monogamous HIV +ve women delivering infected babies. The HIV seroprevalence among children less than 15 years was found to be $7 \%(1.45$ lakhs $) .{ }^{1}$ The vast majority (almost $90 \%$ ) of these new infections in children are believed to stem from Mother to Child Transmission (MTCT). In the absence of interventions, the risk of transmission by an infected mother before or during birth is $15-25 \%$. Breast feeding increases the risk by $5-20 \%$ to a total of $20-45 \% .^{2}$ With specific interventions in nonbreast feeding population, the risk of MTCT can be reduced to less than $2 \%$, and to $5 \%$ or less in breast feeding population. ${ }^{3}$ Use of ART and sdNVP/ syrup NVP to mother-baby pairs has shown to be quite effective in 
preventing this transmission to about $10 \% .4$ It is of paramount importance to reduce the prevalence of paediatric HIV infection and deaths if we want to ensure that the results of reproductive and child health programs are not nullified swiftly.

Alarmed by the rapidly increasing magnitude of the problem in India, the government launched National AIDS Control Organization (NACO) in 1992 for implementation of the project "Prevention of Parent to Child Transmission of HIV/ AIDS (PPTCT)". The discovery of successful interventions to interrupt MTCT has been one of the greatest successes in AIDS research. The challenge in developing countries is to find the most cost-effective and feasible intervention to prevent MTCT.

This need- based study was carried out to emphasize on increasing prevalence of HIV in antenatal patients and also to highlight the fact that with appropriate interventions, MTCT can be reduced significantly among these patients. The main aim of our study was to find out the seroprevalence of HIV in ANC attendees and the incidence of MTCT in HIV +ve pregnant patients using single dose nevirapine (sdNVP) prophylaxis to mothers and neonates as advocated in NACO guidelines.

\section{METHODS}

This prospective cohort study was conducted at Dr. Panjabrao Deshmukh Memorial Medical College (PDMMC) \& Research Centre, Amravati, Maharashtra, from February 2010- May 2015. The main sources of data were detailed proforma filled at the time of admission of the patient, case sheets of the patients and the records at PPTCT center at PDMMC. Data collection was done from Feb 2010 when testing of Dried Blood Spot (DBS) test of neonates for PCR DNA of HIV was started at our center to Nov 2013 till single dose nevirapine was used for preventing MTCT of HIV.

All women attending the antenatal clinic were subjected to pretest counseling by qualified counselors. Those women who were willing underwent HIV testing after written informed consent. If a woman was found to be seropositive for HIV, she was retested using two other kits and declared HIV +ve as per NACO testing (strategy III).

All diagnosed cases (new and old) of HIV + ve pregnant patients during the study period who registered for delivery at Dr. PDMMC were included in the study. The antenatal patients who tested negative for HIV and those HIV +ve patients who did not deliver at PDMMC were excluded from our study. These patients were counseled regarding regular ANC checkup, sdNVP prophylaxis, mode of delivery, safe infant feeding practices and importance of hospital delivery and follow up after delivery. These women were administered sdNVP 200mg at the onset of active phase of labour (minimum $2 \mathrm{hrs}$. before delivery or caesarean section). Caesarean section was done only for obstetric indication. Delivery was carried out with all universal precautions and the neonate was administered nevirapine syrup $(2 \mathrm{mg} / \mathrm{kg}$ body weight) within $72 \mathrm{hrs}$. of birth. The patients who were already on anti-retroviral therapy (ART) were allowed to continue the same during labour and their babies received sdNVP after birth. The choice of infant feeding was according to individual parent's informed decision. The replacement feeding was considered only if AFASS (affordable, feasible, acceptable, sustainable and safe) criteria for exclusive replacement feeding were fulfilled. If patient opted for formula feeding, the method was demonstrated to mother and attendants to ensure safe and hygienic infant feeding. Infants were tested for HIV at 6 weeks, 6 months, 12 months and 18 months of age for detection of perinatal transmission of HIV/ AIDS and were treated accordingly. Results were tabulated and statistical analysis was done.

\section{RESULTS}

A total of 12077 women underwent pretest counselling, of which 11957 were willing to undergo HIV test. Amongst these, 28 were found to be HIV +ve. Twentythree patients opted for delivery at PDMMC, hence were included in the study (Figure 1).

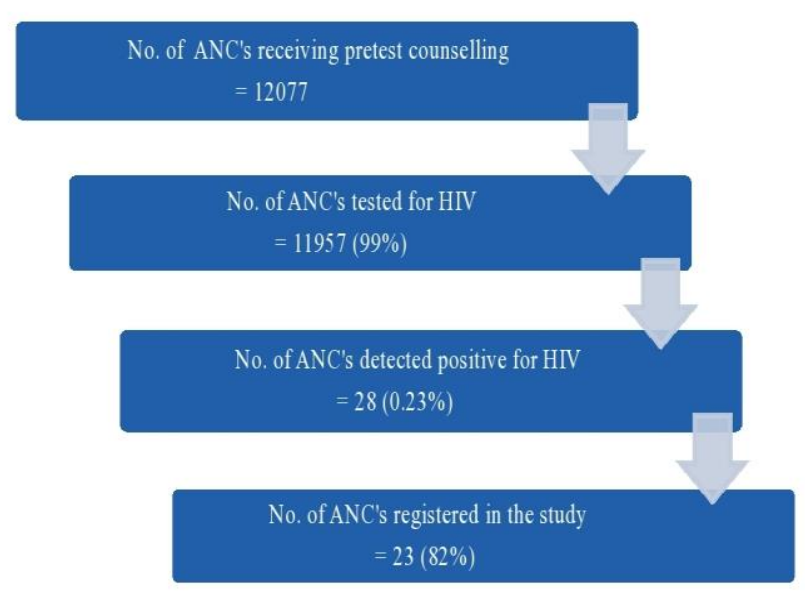

Figure 1: Results of pretest counseling, HIV testing and HIV positive patients registered in the study.

Out of 23 patients, 12 were primigravidae and 11 were multigravidae. Majority of patients were in the age group 20- 29 years, while 3 were aged 30 and above. The husbands of 17 patients were seropositive for HIV (Table 1).

Eight patients were already receiving ART which was continued during pregnancy, labour and after delivery. All patients received sdNVP in active phase of labour and 2 hours before elective LSCS. Fourteen patients delivered between 37- 40 weeks of gestation while 3 patients before 37 weeks and 6 after 40 weeks respectively. 
Fifteen patients delivered vaginally and 8 patients underwent LSCS for various obstetric indications, of which 2 were elective and 6 were emergency LSCS. Birth weight of 15 babies was found to be between 2.1-3.0 $\mathrm{kgs}, 3$ babies weighed $<2 \mathrm{kgs}$ and 5 weighed above 3 kgs. Three low birth weight babies required NICU admission (Table 2).

sdNVP was administered to all babies within 72 hrs. of birth. After counselling regarding infant feeding, 21 patients opted for formula feeding, while 2 opted for breast feeding. Follow up of babies was obtained up to 18 months which revealed 2 babies to be HIV +ve and were started on ART.

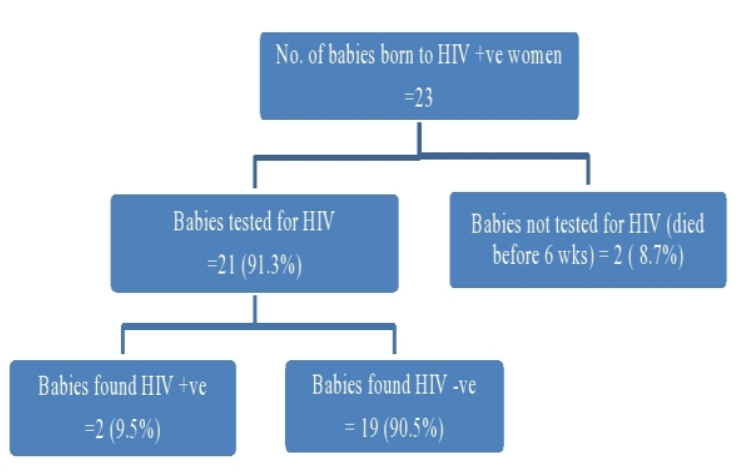

Figure 2: Follow up of babies of HIV positive women.

Table 1: Profile of HIV positive patients $(n=23)$.

\begin{tabular}{|lccc|}
\hline \multirow{3}{*}{ Characteristics } & Category & No. of Patients & Percentage \\
\cline { 2 - 4 } & $20-24$ & 8 & 34.8 \\
\cline { 2 - 4 } & $25-29$ & 12 & 52.2 \\
\cline { 2 - 4 } & $30-34$ & 1 & 4.3 \\
\hline \multirow{2}{*}{ Gravidity } & $>35$ & 2 & 8.7 \\
\hline \multirow{3}{*}{ Husband's HIV status } & Primi & 12 & 52.2 \\
\cline { 2 - 4 } & Multi & 11 & 47.8 \\
\hline & Positive & 17 & 73.9 \\
\hline
\end{tabular}

Table 2: Seropositivity of exposed children in association with mode of delivery, weeks of gestation at delivery, feeding options and ART to mother.

\begin{tabular}{|c|c|c|c|c|}
\hline Factors & Category & Total Babies & Babies Tested For HIV & HIV+Ve Babies \\
\hline \multirow{3}{*}{ Mode of delivery } & Vaginal & 15 & 14 & $2(14.3)$ \\
\hline & Caesarean & 8 & 7 & $0(0)$ \\
\hline & Total & 23 & 21 & $2(9.5)$ \\
\hline \multirow{4}{*}{ Weeks of gestation at delivery } & $<37$ & 3 & 2 & $1(50)$ \\
\hline & $37-40$ & 14 & 13 & $1(7.7)$ \\
\hline & $>40$ & 6 & 6 & $0(0)$ \\
\hline & Total & 23 & 21 & $2(9.5)$ \\
\hline \multirow{3}{*}{ Feeding options } & Formula feeding & 21 & 19 & $2(10.5)$ \\
\hline & Breast feeding & 2 & 2 & $0(0)$ \\
\hline & Total & 23 & 21 & $2(9.5)$ \\
\hline \multirow{3}{*}{ Mother on ART } & Yes & 8 & 7 & $0(0)$ \\
\hline & No & 15 & 14 & $2(14.3)$ \\
\hline & Total & 23 & 21 & $2(9.5)$ \\
\hline
\end{tabular}

*2 babies died before 6 weeks, hence could not be tested for HIV. 
Table 3: Analysis of determinants for the HIV positive babies.

\begin{tabular}{|lll|}
\hline Characteristics & Baby 1 & Baby 2 \\
\hline Mode of delivery & Normal & Normal \\
\hline Gestational at delivery & 40 weeks & 36 weeks \\
\hline Birth weight & $2.2 \mathrm{kgs}$ & $2.6 \mathrm{kgs}$ \\
\hline Mother on ART & No & No \\
\hline Father's HIV status & Positive & Positive \\
\hline Feeding & Formula feeding & Formula feeding \\
\hline
\end{tabular}

2 babies died before 6 weeks hence PCR DNA testing could not be done (Figure 2). One of them was preterm weighing $1.5 \mathrm{kgs}$ and died in NICU after 25 days and second was a full term IUGR baby weighing $2 \mathrm{kgs}$ delivered by emergency LSCS for thick meconium stained liquor. Analysis of the determinants for the 2 seropositive babies is shown in Table 3 .

\section{DISCUSSION}

World Health Organization (WHO) promotes a comprehensive approach for the prevention of HIV infection in infants and young children consisting of four components, the most important being prevention of HIV transmission from mothers living with HIV to their infants. WHO estimates in 2014 state that out of 36.9 million PLHIV, 2.6 million were children with no. of newly infected children being 2.2 lakhs. ${ }^{5} 90 \%$ of all pediatric infections are acquired maternally. PPTCT can be successful only if it is integrated with Maternal \& Child health services.

The most important intervention in the PPTCT program is to enroll as many women for pretest counseling, for which the PPTCT center should be in the premises of antenatal OPD. The second most important step is to enroll maximum no. of women for HIV testing from the pool of pretest counseled patients which was strikingly 99 $\%$ in our study. The seroprevalence rate among ANC attendees in our study was found to be $0.23 \%$. In studies by Mehrotra $\mathrm{R}$ in Allahabad ${ }^{6}$ and Achanta $\mathrm{V}$ et al in Warangal, Andhra Pradesh, ${ }^{7}$ the seroprevalence was $0.4 \%$ and $1.12 \%$ respectively. The spouses of $74 \% \mathrm{HIV}+\mathrm{ve}$ patients were detected seropositive and $26 \%$ were HIVve. Their testing helped in arresting further spread of infection in the community and they were counseled for condom usage.

We followed NACO guidelines to prevent MTCT of HIV by giving sdNVP to mother- baby pairs. No toxicity to nevirapine was observed in mothers and infants. The HIVNET 012 trial in Uganda in 1997-1999 was designed to compare MTCT rates with short course Nevirapine and Zidovudine and it showed HIV infection rates at 18 months as $15.7 \%$ and $25.8 \%$ with both drugs respectively, i.e. sdNVP to mother- baby pairs roughly halved the rate of HIV transmission. Nevirapine has an advantage of long half- life, rapid oral absorption and rapid transplacental passage. The simplicity, efficacy and low cost makes large scale implementation possible even in resource- constrained settings benefitting thousands of babies. Colvin et al also inferred from their study in South Africa that PPTCT programs using sdNVP are effective in reducing the early transmission of HIV to $11.8 \% .^{8}$

$82 \%$ of patients who tested HIV +ve opted for delivery at our hospital. Studies have revealed that vertical transmission during labour is $10-15 \%$. However the route of delivery is to be decided by weighing the risk of HIV transmission against the morbidity due to the intervention. In our study, $65 \%$ patients delivered normally while cesarean section was done for obstetric indications in $35 \%$ patients, of which $25 \%$ were elective. Both the HIV +ve babies belonged to mothers in the normal delivery group. Similar study in Gujarat following NACO guidelines stated reduction in MTCT to $3.6 \%$ with a seroprevalence of antenatal HIV being $0.35 \% .{ }^{9} \mathrm{~A}$ study by Read J S et al proved elective LSCS to be an efficacious intervention for the prevention of MTCT among HIV-1 infected women not taking ARV's or taking only Zidovudine. ${ }^{10}$ Similarly MTCT rates were reduced to $2 \%$ with $\mathrm{ARV}$ prophylaxis during pregnancy, birth and neonatal period along with elective LSCS and avoidance of breast feeding in a randomized clinical trial. ${ }^{11}$ However a study by Shah I in Mumbai proved that efficacy of vaginal delivery along with ART to mother and baby and avoidance of breast feeding is equivalent to that of elective LSCS for MTCT prevention of HIV. ${ }^{12}$

We counseled mothers regarding infant feeding practices and out of 23 patients, only $2(8.7 \%)$ patients opted for breast feeding. The infant feeding options for HIV +ve mothers are replacement feeding with commercial infant formula or home- modified animal milk, wet nursing, exclusive breast feeding with early cessation, expressing and heat treating breast milk and breast milk banks where available. ${ }^{13}$ Whenever the AFASS criteria are fulfilled, use of formula feeding should be preferred as studies have shown that HIV transmission through breast feeding is up to $5-20 \% 2$ and the protective benefit of drugs is diminished when babies continue to be exposed to HIV through breast feeding. A study by Dunn et al states that breast feeding accounts for a $14 \%$ risk of transmission or $0.7-1 \%$ risk per month. ${ }^{14}$ However if patients find artificial feeds to be expensive and availability of safe 
drinking water is limited, then breast feeding is a better option as the risk of malnutrition and life threatening infections like diarrhea and pneumonia may be higher with formula feeding. The famous Thai study in 2004 has proved combination therapy (Zidovudine and lamivudine during last 8 weeks of pregnancy with the addition of sdNVP in labour) has shown to reduce MTCT rates to less than $6 \%$ at 6 weeks in breast- fed infants. ${ }^{15}$

The follow up of babies of HIV +ve patients was excellent at our PPTCT center. Only 2 babies who died before 6 weeks could not be tested. There were no drop outs in the rest of the babies. 2 babies turned out to be HIV +ve in our study. Hence MTCT rate was found to be $9.5 \%$ in this study which is comparable to the rate given in National guidelines by NACO.

sdNVP has been used successfully for PPTCT for almost a decade starting from 2001-02. Though sdNVP significantly reduces the intrapartum HIV transmission; it doesn't cover the risk of transmission during antenatal and breast feeding periods. It also adds to the risk of acquiring drug resistance to nevirapine and other nonnucleoside reverse transcriptase inhibitors (NNRTI). Therefore there has been a global transition to the use of multi- drug Anti Retro Virals (ARV's) to efficiently bring down the rate of MTCT to less than 5\%. Thus "National Guidelines for PPTCT in India (2013)" have been revised to incorporate recommendations given by WHO (June 2013) which states that every HIV patient receives ART/ ARV prophylaxis starting as early as 14 weeks irrespective of her CD4 counts which is continued during intrapartum and postpartum period. Babies of all HIV +ve women are administered nevirapine syrup $(2 \mathrm{mg} / \mathrm{kg})$ for 6 weeks. We plan to extend our study following the new protocol so as to compare the rates of MTCT with the old and the new regimens.

\section{CONCLUSIONS}

In developing countries like India where access to even basic antenatal care is sometimes unavailable, universal screening for HIV is a great challenge. The lack of adequate knowledge regarding HIV makes it extremely difficult to eliminate myths and misunderstandings among pregnant women. Health education and awareness campaigns should target antenatal patients as in spite of seroprevalence being low in this group, the acceptability of interventions aimed at MTCT prevention is higher during this period. The seroprevalence in our study was $0.23 \%$. Universal screening of pregnant patients for HIV and timely intervention would result in a significant decline in paediatric HIV infection.

With rapid globalization of HIV research, recommendations for PPTCT have kept changing over the last few years. Studies conducted to test use of single or combination drugs for MTCT prevention; route of delivery (normal or caesarean) and type of infant feeding (breast feeding or formula feeding) have given varied results depending on various factors in target population. Hence choice of judicious PPTCT regimen suited to Indian setting would result in significant reduction in MTCT of HIV in our country. We could reduce MTCT to 9.5\% using sdNVP regimen. The contributing factors behind the success of our study were excellent pretest counselling by PPTCT staff members leading to $99 \%$ patients undergoing HIV testing, all mother- baby pairs receiving sdNVP, $91 \%$ mothers opting for formula feeding and no drop- outs in the follow up of infants of HIV + ve mothers. The inclusion of more effective ART regimens in NACO guidelines will further reduce the perinatal transmission of HIV. We should strictly follow these guidelines for the successful implementation of PPTCT program and to help India move closer to the global target of "Zero new infections and zero AIDSrelated deaths".

\section{ACKNOWLEDGEMENTS}

We are thankful to Dr Pushpa Junghare, HOD, Department of OBGY and ICTC incharge, Dr. PDMMC Amravati, for allowing us to publish this data. We are also grateful to Dr. Pushpa Lokare, Associate Professor, Department of Community Medicine, Dr. PDMMC Amravati, for helping us with the statistical analysis. We highly appreciate the efforts of Mrs. Priti Hande, counsellor, and Mrs Varsha Gharde, technician, PPTCT centre of our college for keeping a relentless follow up of patients and for excellent maintenance of records without which this work would have been impossible.

Funding: No funding sources

Conflict of interest: None declared

Ethical approval: The study was approved by the Institutional Ethics Committee

\section{REFERENCES}

1. NACO Annual Report 2013-14. Department of AIDS control, Ministry of Health and Family Welfare, Government of India. http://www.naco.gov.in/upload/2014mslns/NACO_E nglish 2013-14.pdf. Accessed 25 July 2015.

2. WHO. PMTCT Strategic Vision 2010-15. Preventing mother- to- child transmission of HIV to reach the UNGASS and Millennium Development Goals. http://www.who.int/hiv/pub/mtct/strategic_vision.pdf . Accessed 1 Aug 2015.

3. WHO, UNICEF, UNAIDS, UNFPA. HIV transmission through breast feeding- a review of available evidence, update 2007. http://whqlibdoc.who.int/publications/2008/9789241 596596_eng.pdf. Accessed 18 June 2015.

4. NACO. Updated National guidelines for prevention of parent- to- child transmission of HIV using Multi drug Anti- Retroviral regimen in India. Issued in Dec. 2013. http: //naco.gov.in/upload/NACPIV/18022014BSD/National_Guidelines_for_PPTCT. pdf. Accessed 12 July 2015. 
5. WHO, UNICEF, UNAIDS, HIV department. Global summary of AIDS epidemic, 2014. Issued on 21st July,

2015 . http://www.who.int/hiv/data/epi_core_july 2015.png? ua=1. Accessed 27 July 2015.

6. Mehrotra R, Pourush S, Bhargava A, Verma M, Ghosh U K. Seroprevalence of HIV in antenatal women. J Obstet Gynecol India. 2005;55:333-5.

7. Achanta V, Sirikki P C, Madisetty V S. Five years of parent to child transmission of HIV/ AIDS program in a rural based teaching hospital. J Obstet Gynecol. 2011;61:275-9.

8. Colvin M, Chopra M, Doherty T, Jackson D, Levin $\mathrm{J}$, Willumsen $\mathrm{J}$, et al. Operational effectiveness of single- dose nevirapine in preventing mother- tochild transmission of HIV. Bulletin of WHO; 85:466-73.

9. Joshi U, Kadri A, Bhojiya S. Prevention of parent to child transmission services and interventionscoverage and utilization: A cohort analysis in Gujarat, India. Indian $\mathbf{J}$ Sex Transm Diseases 2010;31(2):92-8.

10. Read JS, Newell ML. Efficacy and safety of cesarean delivery for prevention of mother- to- child transmission of HIV- 1. (Cochrane review). In: The Reproductive Health Library, Issue 9, 206. Oxford: Update Software Ltd. Available from http://www.rhlibrary.com.
11. The European Mode of Delivery Collaboration. Elective cesarean section versus vaginal delivery in prevention of vertical HIV- 1 transmission; a randomized clinical trial. Lancet 1999; 353(9158):1035-9.

12. I Shah. Is elective cesarean section really essential for prevention of MTCT of HIV in the era of ART and abstinence of breast feeding? J Trop Pediatrics, 2006;52(3):163-5.

13. WHO, UNICEF, UNFPA, UNAIDS. HIV and infant feeding- A guide for health care managers and supervisors. Issued in 2003. http://www.who.int/nutrition/publications/HIV_IF_g uide_for_healthcare.pdf. Accessed 21 July 2015.

14. Dunn D, Newell M, Ades A E, Peckham C S. Risk of HIV-1 transmission through breast feeding. Lancet 1992;340(8819):585-8.

15. Lallemant M, Jourdain G, Le Coeur S, Mary J Y, Ngo-Giang-Huong N, Koetsawang $\mathrm{S}$ et al. Single dose perinatal nevirapine plus standard zidovudine to prevent mother- to- child transmission of HIV- 1 in Thailand. New England Journal of Medicine 2004;351:217-28.

Cite this article as: Bhuyar SA, Kale PV.

Seroprevalence of human immunodeficiency virus among antenatal patients and incidence of motherto- child transmission using single dose nevirapine: a cohort study in Maharashtra. Int J Reprod Contracept Obstet Gynecol 2015;4:1436-41. 